\title{
The effect of shape and location on temporal masking of spatial vibrotactile patterns
}

\author{
DAVID T. HORNER \\ University of Wisconsin, Oshkosh, Wisconsin
}

\begin{abstract}
Target patterns presented to uncued locations on a single fingerpad were followed by either sameshape (SS) maskers or different-shape (DS) maskers presented to either the same location (SLoc) or a different location (DLoc). DS maskers interfered with identification more when they were at SLoc than when they were at DLoc, but the reverse was true for SS maskers; they interfered more at DLoc than at SLoc. When targets were presented to cued locations, performance in the absence of maskers improved, but the pattern of interference from maskers resembled that for uncued presentation. The proportion of masker responses on incorrect trials revealed that both temporal masking and response competition may be involved in the effects of location on pattern identification.
\end{abstract}

The present study examined whether the location of a pattern on the skin affects the ability to process information about its spatial features. Specifically, does location affect the ability to identify spatial patterns on a single fingerpad? The fingerpad was examined because of its high spatial resolution (Sathian \& Zangaledze, 1996; Sherrick \& Cholewiak, 1986; Stevens \& Patterson, 1995; Van Boven \& Johnson, 1994; Weinstein, 1968), because of its role in tactual exploration, and because it is used to process successive spatial patterns in tactile communication systems such as braille and the Optacon (Bliss, Katcher, Rogers, \& Shepard, 1970). In studies using the Optacon vibratory array or similar dense arrays of vibrators, spatial patterns have usually been presented either to a single location or to locations on separate body sites. Some of the studies examining separate body sites have compared the pattern processing capabilities of the sites (Cholewiak \& Craig, 1984; Craig, 1977; Loomis, 1980; Scadden, 1973). In other studies examining separate sites, patterns were presented to separate fingers and attentional factors were investigated (Craig, 1985a; Craig \& Evans, 1995; Evans \& Craig, 1991, 1992; Evans, Craig, \& Rinker, 1992; Horner, 1992, 1995; Rinker \& Craig, 1994). Relatively few studies have examined how spatial patterns are processed at adjacent locations on a single fingerpad.

Hill (1974) indirectly measured the effects of presenting patterns to adjacent locations on the fingerpad when

\footnotetext{
An earlier version of some of the material in Experiment 1 was presented at the annual meeting of the Psychonomic Society in November 1993. An earlier version of some of the material in Experiment 2 was presented at the annual meeting of the American Psychological Society in June 1995. This research was supported by the University of Wisconsin, Oshkosh, and by Grant DC-00095 from the National Institutes of Health. D.T.H. wishes to thank James Craig for his comments on earlier versions of this manuscript and Roger Rhodes for his assistance in conducting these experiments. Correspondence should be addressed to D. T. Horner, Psychology Department, University of Wisconsin, Oshkosh, WI 54901-8601 (e-mail: horner@vaxa.cis.uwosh.edu).
}

he examined Optacon reading rates using different display conditions. He presented letters on the display moving up or down the fingerpad and measured reading rates for full displays and for half displays to determine whether observers made use of an increased tactile field of view. He found that reading rates were slower using the bottom half than the top half of the display, and thus adjacent locations differed in their ability to process spatial patterns. Nolan and Kederis (1969) found that the locations of dots in braille characters affected recognition; lower cell characters were recognized more slowly than were upper cell characters. In addition, dots at the bottom of the cell were missed more frequently than those at the top. Nolan and Kederis suggested that these differences between locations may have been due to perceptual and attentional factors as well as the relative frequency of occurrence of upper and lower cell characters in braille reading material.

Horner (1995) used a discrimination task to directly examine whether the locations of patterns on the fingerpad affected the ability to process information about their shapes. Pairs of patterns were presented sequentially either to identical locations or to locations separated by various distances. Observers were asked to ignore location and, on the basis of shape alone, to respond "same" or "different" to pairs of same-shape (SS) patterns or different-shape (DS) patterns. Discrimination performance declined with increasing distance between patterns despite the fact that location was experimentally defined as an irrelevant attribute, suggesting that observers were unable to process shapes of patterns independently of location. The representations of patterns at separate locations may have required alignment to compare the amount of overlap between patterns.

The effect of location on discrimination may have been partly due to the nature of the task. Discrimination involves comparing the features of two patterns, and presenting the patterns to separate locations might interfere with feature comparison. If no such comparison were required, 
the effects of location might be much reduced or perhaps even eliminated. Horner (1995) used a discrimination measure partly because it required processing pairs of patterns presented sequentially, a task that resembles the way tactile patterns are processed as the fingers move over an object's surface. However, when inspecting objects tactually, observers are often required to identify individual features on the object's surface. As the fingers are moved along the surface, features contact the skin sequentially, and identifying an individual feature may require separating it from temporally preceding or following stimuli-a temporal masking task. Unlike discrimination tasks, temporal masking tasks involve identifying a single member of a pair of patterns, the target. Observers attempt to ignore the other member of the pair, the masker.

In tactual exploration of objects, separate surface features may contact different locations on a single fingerpad, and identifying a feature at one location may be affected by features at other locations. If shape information is independent of location, varying the locations of targets and maskers may have little effect on target identification. The term independent used in the present study refers to whether or not changing the locations of targets and maskers, while keeping their shapes constant, alters target identification. Since the discriminability of tactile patterns is affected by varying their distance, it is likely that target identification will be affected by the locations of maskers. A study on the perceived similarity of tactile patterns suggested that information about shape may not be independent of location (Weisenberger, 1981). Observers identified targets followed by DS maskers at the same location as the target or at an adjacent location on the same fingerpad. Maskers interfered with target identification to a greater extent when they were at the same location than when they were at an adjacent location. Because the study focused on perceived similarity, it did not systematically compare the effects of shape and location of maskers.

In the present study, pairs of SS and DS patterns were presented sequentially to either identical locations or separate locations on the same fingerpad. Observers were asked to ignore the locations of the patterns and to identify the target and ignore the masker. The question was whether varying the shape and location of the masker would have independent effects on target identification. Evans (1987) presented targets and maskers sequentially to the same location and found that SS maskers interfered little with target identification, whereas DS maskers interfered greatly, due to temporal integration of target and masker features. Craig and Evans (1987) also examined the effects of presenting SS or DS maskers to the same location as the target. However, they focused on the temporal persistence of maskers and not the effects of presenting such maskers adjacent to the target location. The present study focused primarily on identification performance under the combined effects of varying masker shape and location.
Identifying targets in the presence of SS or DS maskers may depend not only on location, but also on information about where the target is located. For example, when moving the fingers over an object's surface, observers may be unaware of the impending contact of features at specific locations, but after contacting such features, the fingerpad may be repositioned to feel the features at specific, known locations. Before contacting the features, observers have no knowledge of which locations the features will occupy, but after contact, these locations are known. One might expect better identification with knowledge of the locations of features, but identification may also be possible without a priori knowledge of their locations. An additional goal of the present study was to compare identification performance when observers had foreknowledge of the target's location and when they had no such knowledge. Such a comparison provides a measure of the ability to attend selectively to adjacent locations on a single fingerpad.

Attention has been described as a "spotlight" highlighting a region of space that enjoys special processing capabilities (Driver \& Baylis, 1991; Eriksen \& Hoffman, 1973). Stimuli within the spotlight of attention are processed more quickly and accurately than are stimuli outside the spotlight. Furthermore, the space "illuminated" by the attentional spotlight may vary in size depending on task requirements. Previous studies of tactile pattern perception have not examined the extent of spatial attention on a single fingerpad. In Experiment 1, targets and maskers were presented randomly to one of two locations, and observers did not know which location the target would occupy (uncued presentation). If observers maintain a wide attentional spotlight to cover both locations, they may have difficulty identifying the target. They may also be unable to avoid processing maskers at adjacent locations, leading to interference in target identification. In Experiment 2, performance during uncued presentation was compared with performance when observers knew where the target would appear (cued presentation). If observers can attend selectively to a single location, cued performance should exceed uncued performance. Furthermore, if observers narrow their attentional spotlight to a single location during cued presentation, they may avoid processing adjacent maskers, which would lead to reduced interference.

\section{EXPERIMENT 1}

Experiment 1 directly compared the effects of presenting SS and DS maskers either to the same location (SLoc) as the target or to a different location (DLoc) than the target. DS maskers should interfere with target identification more when they are presented to SLoc than to DLoc, and there should be little interference when SS maskers are presented to SLoc. Horner (1995) found that pairs of SS patterns presented to separate locations on a single fingerpad often feel like DS patterns, suggesting that SS maskers presented to DLoc may interfere with 
target identification. Furthermore, target localization is disrupted by the presence of identical stimuli at separate locations (Craig, 1989). To the extent that SS maskers at DLoc feel like DS maskers, they may interfere with target identification in a manner similar to that of DS maskers. If SS maskers at DLoc interfere with target identification, this would support the view that location is a distinctive feature of tactile patterns. If so, then despite the fact that pattern shapes are identical at separate locations, the additional factor of location may be important for perception. Furthermore, evidence that location plays an important role in perceiving the shapes of patterns would be provided from two separate measures, discrimination (Horner, 1995) and identification. This type of "converging evidence" is an important source of the validity of research findings (Campbell \& Fiske, 1959).

Previous temporal masking studies have varied the time between target and masker onsets-stimulus onset asynchrony (SOA) - in order to examine the temporal characteristics of pattern processing (Cholewiak \& Craig, 1984; Craig, 1976, 1980, 1982, 1983, 1985a; Craig \& Evans, 1987; Evans, 1987; Evans \& Craig, 1986; Horner, 1991; Horner \& Craig, 1989). SOA was varied in Experiment 1 to determine the extent to which the locations of patterns affected the rate at which information was processed. Maskers typically interfere with target identification more at brief SOAs than at long SOAs (Cholewiak \& Craig, 1984; Craig, 1976, 1978, 1982, 1983, 1985a; Craig \& Evans, 1987; Evans, 1987; Evans \& Craig, 1986; Horner, 1991; Horner \& Craig, 1989). Thus, the greatest effects of varying the shapes and locations of targets and maskers should occur over relatively brief time intervals. At longer intervals, varying shape and location may have less effect on target identification.

\section{Method}

Participants. Six observers were tested, all of whom had received practice identifying letter-like patterns. One male and 4 female students were paid to participate and the 6th observer was D.T.H. One of the 6 observers (not the author) performed at chance level, and the data from this observer were therefore excluded from the analysis.

Apparatus. A PDP-11/34 computer controlled the amplitude and duration of vibration of the individual pins in the vibratory array of the Optacon, a reading aid for the blind (Bliss et al., 1970). The vibratory array measures $11.45 \mathrm{~mm}$ wide $\times 27.14 \mathrm{~mm}$ high and consists of 144 blunt pins arranged in 24 rows $(1.18 \mathrm{~mm}$ separating rows) and six columns ( $2.29 \mathrm{~mm}$ separating columns). Each of the .25-mm-diameter pins vibrated at $230 \mathrm{~Hz}$ and at a comfortable intensity well above threshold, $33 \mathrm{~V}$ to the driver circuits, resulting in a maximum skin indentation of 65 microns (Bliss et al., 1970; Bliss \& Linvill, 1966). The vibratory array contacted the distal portion of the left index fingerpad.

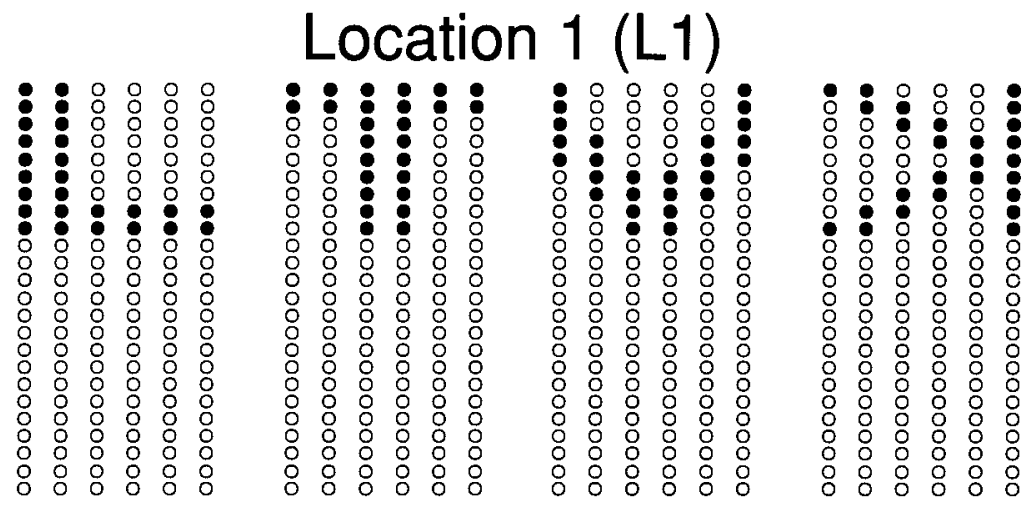

\section{Location 2 (L2)}


Figure 1. Representations of the patterns used as targets and maskers in Experiment 1 at each of the two array locations where the patterns were presented. Filled circles indicate activated pins in the array and unfilled circles indicate inactive pins. 
Stimuli. Targets and maskers were randomly selected with replacement from the same set of stimuli on each trial. The stimuli were patterns shaped like an $\mathrm{L}$, a $\mathrm{T}$, a V, and a reverse $\mathrm{K}$ (Figure 1). Each pattern was presented for $26 \mathrm{msec}$ in a "static" mode in which all of the pins in the pattern were activated simultaneously (Craig, 1980). As shown in Figure 1, each pattern occupied an area on the array six columns $\times$ nine rows, or $11.45 \mathrm{~mm}$ wide $\times 9.44 \mathrm{~mm}$ high. In addition, the patterns were activated randomly at one of two locations on the array: rows $1-9$ (Location 1 [L1]), or rows 10-18 (Location 2 [L2]). On any given trial, the target and masker could occupy identical locations, or the centers of the patterns could be separated by 10 rows $(10.62 \mathrm{~mm})$. Pins in rows $19-24$ remained inactive. The total area covered by both locations ( $\mathrm{L} 1$ and $\mathrm{L} 2$ ) was $11.45 \mathrm{~mm}$ wide $\times 20.06 \mathrm{~mm}$ high.

Procedure. Observers were seated with their left index fingers on the tactile array in front of them. Pictures of the patterns in Figure 1 were placed above the first four number keys on a standard IBM keyboard. Observers initiated trials and responded with their right hands by pressing the appropriate keys. Observers wore earplugs and earphones emitting low-pass, filtered noise to eliminate auditory information. Observers were told on each trial that a pair of patterns would be presented sequentially, and that each pattern would be presented randomly at one of two locations, near the top or bottom of the distal part of the fingerpad. Observers were told to identify the first pattern, the target, on the basis of its shape, ignoring where the pattern was felt on the fingerpad, and to ignore the second pattern, the masker. Observers were told nothing else about the maskers.

On each trial, observers received a cue stimulus consisting of the first and last pins in the top two rows, activated for $13 \mathrm{msec}$. The cue stimulus was also available at the observer's command between trials and served to ensure that the finger was correctly positioned on the array. One second after the cue stimulus, the target was presented randomly to one of the two locations, followed at an SOA of either 75 or $150 \mathrm{msec}$ by the masker, which was also presented randomly to one of the two locations. A third condition in which the masker was absent was also tested. Trial-by-trial feedback was provided, consisting of either the word correct appearing on a visual display in front of the observer, or, when the response was incorrect, the number corresponding to the correct choice. Response times (RTs) were also measured on each trial: Observers were told to respond as quickly as possible without sacrificing accuracy for speed. Accuracy was therefore emphasized, and no RT feedback was provided. Trials were presented in blocks of 90 , with each block testing a different condition (two SOA conditions and a masker-absent condition) in random order. Data were collected over six sessions ( 540 trials per SOA condition for each observer).

\section{Results and Discussion}

The percentage of correct target responses (PC) and the mean RT on correct trials were computed for each observer. Trials with RTs below 300 or above $2,500 \mathrm{msec}$ were excluded from the analysis. As in previous tactile pattern perception studies, $\mathrm{PC}$ was the main measure of performance and RTs were recorded primarily to determine whether the results might be due to a speed-accuracy tradeoff(Craig \& Evans, 1995; Evans \& Craig, 1991, 1992; Evans et al., 1992; Horner, 1995). No speed-accuracy tradeoff was evident. As in previous studies, an inverse relationship existed between RT and PC; higher accuracy was associated with lower RTs. Averaged across observers, $\mathrm{PC}$ in the absence of a masker was significantly higher at L1 (76\%) than at L2 $(61 \%)[t(4)=3.24, p<$ $.05]$, indicating a processing advantage at $\mathrm{L} 1$. This result may have been due to differences in the density of innervation of first-order afferents at the two locations (Johansson \& Vallbo, 1979; Vallbo \& Johansson, 1984).

$\mathrm{PC}$ in the presence of maskers was analyzed according to the shapes and locations of the target and masker on each trial. Because of the mean difference in performance at L1 and L2, the data were analyzed separately for each location. Figure 2 shows PC averaged across observers in the presence of SS or DS maskers at SLoc or DLoc as a function of SOA. Also shown is PC in the absence of a masker. Each data point on the SS functions represents at least 150 trials, and each data point on the DS functions represents at least 469 trials. The data for individ-



Figure 2. Percentage of correct target responses as a function of stimulus onset asynchrony (SOA). The left panel shows performance when targets were at $L 1$ and the right panel shows performance when targets were at $L 2$. The functions in each panel show performance when SS maskers were at SLoc (SS-SLoc), when SS maskers were at DLoc (SS-DLoc), when DS maskers were at SLoc (DS-SLoc), and when DS maskers were at DLoc (DS-DLoc). L, location; SS, same shape; DS, different shape. 
ual observers were similar to those shown in Figure 2: The SEMs for each data point ranged from $2 \%$ to $12 \%$ and did not differ systematically across SOA, masker shape, or masker location. The data for L1 and L2 are discussed separately in the following sections.

Target at L1. The L1 data were analyzed using a $2 \times$ $2 \times 2$ (masker shape $\times$ masker location $\times$ SOA) repeated measures analysis of variance (ANOVA). Only the main effects and interactions reaching at least the .05 level of significance are discussed here. There was a main effect of masker shape, showing that accuracy was lower in the presence of DS than of SS maskers $[F(1,4)=$ $20.95, p<.02]$. This differential effect of masker shape was greater at the brief SOA than at the long SOA, as indicated by an interaction between masker shape and SOA $[F(1,4)=10.67, p<.05]$. Performance in the presence of DS maskers improved with increasing SOA, but little improvement occurred for SS maskers.

Many studies have shown that performance improves with increasing SOA when DS maskers are at SLoc (Cholewiak \& Craig, 1984; Craig, 1976, 1978, 1982, 1983, 1985a; Craig \& Evans, 1987; Evans, 1987; Evans \& Craig, 1986; Horner, 1991; Horner \& Craig, 1989). Such improvement may be due to several factors, including a decline in temporal integration of target and masker features at longer SOAs (Evans, 1987; Evans \& Craig, 1986) which would improve the clarity of individual features within the target. If the masker and target are identical, temporal integration of their features should cause little interference, and this was the case for SS maskers at SLoc. Performance also improves with increasing SOA in studies of tactile metacontrast in which DS maskers surround the target (Weisenberger \& Craig, 1982). In the present study, DS maskers at DLoc may have interfered at brief SOAs because of their spatial proximity to the target, a type of lateral masking effect (Loomis, 1990, 1993; Loomis \& Apkarian-Stielau, 1976), and such an effect might disappear at longer SOAs. In addition, performance improved at similar rates whether DS maskers were at SLoc or DLoc, consistent with the fact that time courses of tactile metacontrast functions resemble those of recognition masking functions (Weisenberger \& Craig, 1982).

The $\mathrm{L} 1$ data also showed an interaction between masker shape and masker location $[F(1,4)=23.65, p<.05]$ : DS maskers interfered more at SLoc than DLoc, whereas SS maskers interfered more at DLoc than SLoc. Greater interference by DS maskers at SLoc than at DLoc may have been due to temporal integration of target and masker features at SLoc and temporal order being the only cue as to which pattern was the target. For DS maskers at DLoc, location was an additional cue for determining which was the target pattern, perhaps partly accounting for reduced interference. Interference by SS and DS maskers at DLoc may have been due to lateral masking (Loomis, 1990, 1993; Loomis \& ApkarianStielau, 1976).

An alternative explanation for interference is that observers were often unable to restrict their attention to a single location and sometimes processed both maskers and targets to the point of incipient response activation (Evans \& Craig, 1992; Evans et al., 1992). DS maskers might have activated responses that competed with target responses. Such response competition may have been more likely for maskers at SLoc than at DLoc, and more likely at the brief than the long SOA. SS maskers at SLoc would have activated the same response as the target and produced no interference, as was the case. However, SS maskers at DLoc were at L2 and may have been relatively difficult to perceive or may have been perceived as different shapes than the target and activated different (nontarget) responses, leading to interference. The possibility of response competition is explored further in the next section.

Target at L.2. Statistical analysis of the L2 data produced results similar to those of the $\mathrm{L} 1$ data except for one important difference. There was no interaction between masker shape and masker location $[F(1,4)=.08$, $p>.05]$ because DS maskers produced similar interference at SLoc and DLoc, and SS maskers produced little interference at both SLoc and DLoc. Response competition may explain the $\mathrm{L} 2$ results. Since patterns were easier to recognize at $L 1$ than at $L 2$, maskers at $L 1$ may have activated stronger responses than maskers at L2. If so, DS maskers at L1 may have interfered more with target identification than maskers at L2. This appears to be the case; the two lowest functions in Figure 2 correspond to maskers at L1. SS maskers at L1 should have interfered little because responding with an SS masker would produce a correct response, and this was also the case.

Although Experiment 1 was not designed to differentiate between possible causes for interference (e.g., response competition vs. masking), it is possible to examine the role of response competition indirectly by determining how often observers responded with the masker instead of the target. To estimate how often observers may have responded with maskers, the data on incorrect DS masker trials were analyzed across SOA to determine the percentage of masker responses. If observers had no knowledge of the masker, they would have responded with the masker on $33 \%$ of the incorrect trials by chance alone. When DS maskers were at L1, observers responded with the masker on $52 \%$ of the incorrect trials. When DS maskers were at $\mathrm{L} 2$, observers responded with the masker on $46 \%$ of the incorrect trials. Clearly, observers responded with the masker more of ten than chance. However, observers responded only slightly more often when the masker was at $\mathrm{L} 1$ than at L2. It is unclear whether this difference in responding with the masker based on its location accounts for the different pattern of results when targets were at $\mathrm{L} 1$ and $\mathrm{L} 2$.

Masker responses occurred more often than chance whether the masker was at L1 or L2, suggesting that observers were unable to restrict attention to a single location. Studies of tactile response competition have shown that observers are unable to restrict attention to targets on one fingerpad when nontargets with a competing response are presented to an adjacent fingerpad (Craig \& 
Evans, 1995; Evans \& Craig, 1991, 1992; Evans et al., 1992; Rinker \& Craig, 1994). Failure of selective attention in these types of tasks (but not necessarily in other tasks; see Sathian \& Burton, 1991, and Whang, Burton, \& Shulman, 1991) occurs over temporal intervals of as long as $150 \mathrm{msec}$ between patterns--that is, equal to the longest SOA examined in Experiment 1. Furthermore, Craig and Evans (1995) showed that the shape of the function relating performance to SOA is similar whether nontargets are presented to adjacent fingers or to the target location. It is therefore not too surprising that observers in Experiment 1 often responded with the masker.

Although the results of Experiment 1 are consistent with a response competition explanation, temporal integration of target and masker features may have occurred when maskers were at SLoc, especially at the brief SOA. Craig (1995) has shown that interference between successive patterns is sometimes due to a combination of temporal masking and response competition. Furthermore, some of the present results appear to be consistent with a lateral masking explanation. A variety of factors may be involved in the effects of location on identification. Experiment 2 explored these issues further.

\section{EXPERIMENT 2}

Experiment 2 compared uncued presentation with performance when observers were informed of the target's location before each trial (cued presentation). Cued presentation is similar to the situation encountered by Optacon users and braille readers who know ahead of time where patterns are located. The primary goal of Experiment 2 was to determine whether knowledge of the target's location would improve performance by allowing observers to focus attention on a single location. In Experiment 1 , observers may have used a wide attentional spotlight, leading to interference by maskers at DLoc via response competition. If so, narrowing attention to a single location might reduce interference by maskers at DLoc in the cued condition relative to the uncued condition. Although there may be an advantage in directing attention to a single finger over dividing attention between fingers (Craig, 1985b; Sathian \& Burton, 1991; Whang et al., 1991), no studies have examined whether there is a similar advantage in directing attention to one of two possible locations on a single fingerpad.

Experiment 2 also indirectly assessed the role of factors occurring at a relatively early stage in processing. Craig (1995) suggested that when both target and masker representations are available to observers, response selection may be improved by providing observers information about the sequence of patterns to be presented. Informing observers of the target's location before each trial may improve response selection, leading to a reduction in the proportion of masker responses. If Experiment 2 eliminates overresponding with the masker, any remaining interference should be due to factors occurring at a relatively early stage in target processing. Because observers should be less likely to respond with maskers the longer the SOA, the longest SOA tested was increased to $220 \mathrm{msec}$. Because Experiment 1 used an uncued presentation method, the uncued condition in Experiment 2 provided an opportunity to determine whether the results of Experiment 1 were limited by the specific pattern set used. Therefore, in Experiment 2 the number of patterns was reduced to three and different shapes were used to ensure the generality of the results.

\section{Method}

Participants. Six initially naive observers were tested. Four male and 2 female students were either paid or received course credit for participation.

Apparatus. An IBM PS2/ValuePoint 466DX2 computer was interfaced with the vibratory array from the Optacon II, a newer version of the Optacon used in Experiment 1. The Optacon II array measures $9.6 \mathrm{~mm}$ wide $\times 22.8 \mathrm{~mm}$ high and consists of 100 blunt pins arranged in 20 rows ( $1.2 \mathrm{~mm}$ separating rows) and 5 columns ( $2.4 \mathrm{~mm}$ separating columns). Each of the .25 -mm-diameter pins vibrated at $230 \mathrm{~Hz}$ and at a comfortable intensity well above threshold. The vibratory array contacted the distal portion of the left index fingerpad.

Stimuli. Targets and maskers were randomly selected with replacement from the same set of three patterns on each trial (Figure 3). Each pattern was presented in the static mode for $70 \mathrm{msec}$. As shown in Figure 3, each pattern occupied an area on the array that was five columns $\times$ nine rows, or $9.6 \mathrm{~mm}$ wide $\times 9.6 \mathrm{~mm}$ high, at either L1 or L2. Either targets and maskers occupied identical locations, or the centers of the patterns were separated by 10 rows $(10.8 \mathrm{~mm})$. The total area covered by both locations $\mathrm{Ll}$ and L2 was $9.6 \mathrm{~mm}$ wide $\times 20.4 \mathrm{~mm}$ high.

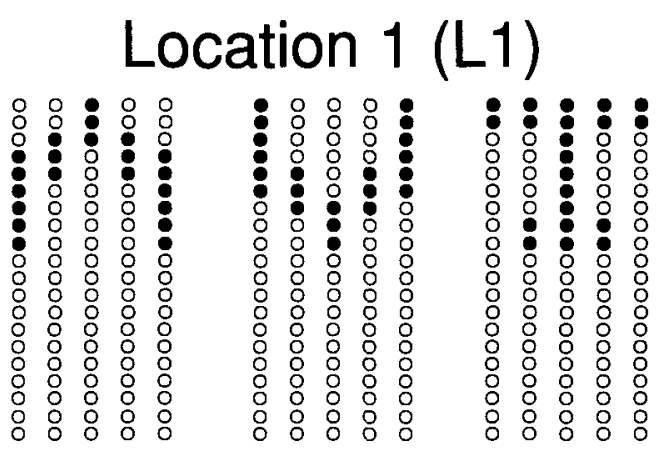

\section{Location 2 (L2)}
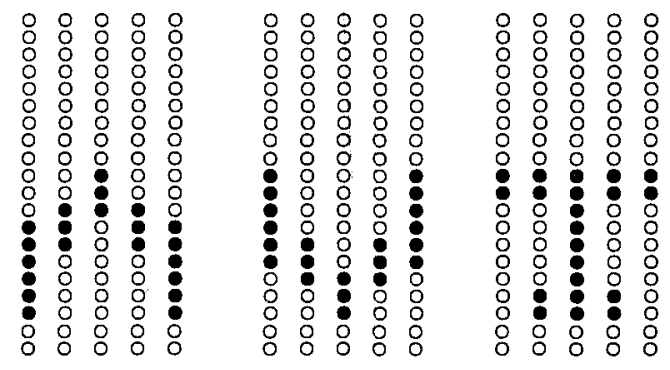

Figure 3. Representations of the patterns used as targets and maskers in Experiment 2 at each of the two array locations where the patterns were presented. Filled circles indicate activated pins in the array and unfilled circles indicate inactive pins. 
Procedure. The same procedure was used as that in Experiment 1 except for the following changes. There was no initial cue stimulus on each trial because shorter blocks of trials were used and because the cue stimulus was available between trials. Because the stimuli were of longer duration, longer SOAs (130 and $220 \mathrm{msec}$ ) were used. RT was not measured, and observers were told to focus only on accuracy. Before each trial, observers were either informed of the target's location (cued presentation) or not informed (uncued presentation). Maskers followed the targets randomly at one of the two locations. Cued trials were blocked by target location and SOA, and uncued trials were blocked only by SOA. Three blocks of trials in which the masker was absent were included; one uncued block and two cued blocks (one for each location). Thus, each session contained nine 30-trial blocks in random order. Data were collected over 10 sessions ( 300 trials per SOA for each observer).

\section{Results and Discussion}

$P C$ in the absence of a masker was averaged across observers and the data were analyzed using a $2 \times 2$ (presentation type $\times$ target location) ANOVA. PC in the absence of a masker was higher for cued $(M=79 \%)$ than for uncued $(M=70 \%)$ presentation $[F(1,5)=12.14$, $p<.02$ ], suggesting that observers may have narrowed attention to a single location in the cued condition. Thus, there appears to be an advantage in directing attention to one of two possible locations on a single fingerpad. The difference between cued and uncued performance is especially noteworthy given that in the uncued condition, observers are presumably either attending to both locations simultaneously or alternately directing attention to a single location. PC in the absence of a masker was also higher at $\mathrm{L} 1$ than at $\mathrm{L} 2[F(1,5)=28.42, p<.01]$. There was no significant interaction between presentation type and target location.

PC in the presence of SS or DS maskers at SLoc or DLoc was analyzed separately for each target location, as in Experiment 1. Performance averaged across observers is shown in Figure 4 as a function of SOA separately for each presentation condition, along with $\mathrm{PC}$ in the $\mathrm{ab}$ sence of a masker. Each data point on the SS functions represents at least 140 trials, and each data point on the DS functions represents at least 278 trials. The data for individual observers were similar to those shown in Figure 4: The $S E M$ s for each data point ranged from $2 \%$ to $8 \%$ and did not differ systematically across SOA, masker shape, or masker location.

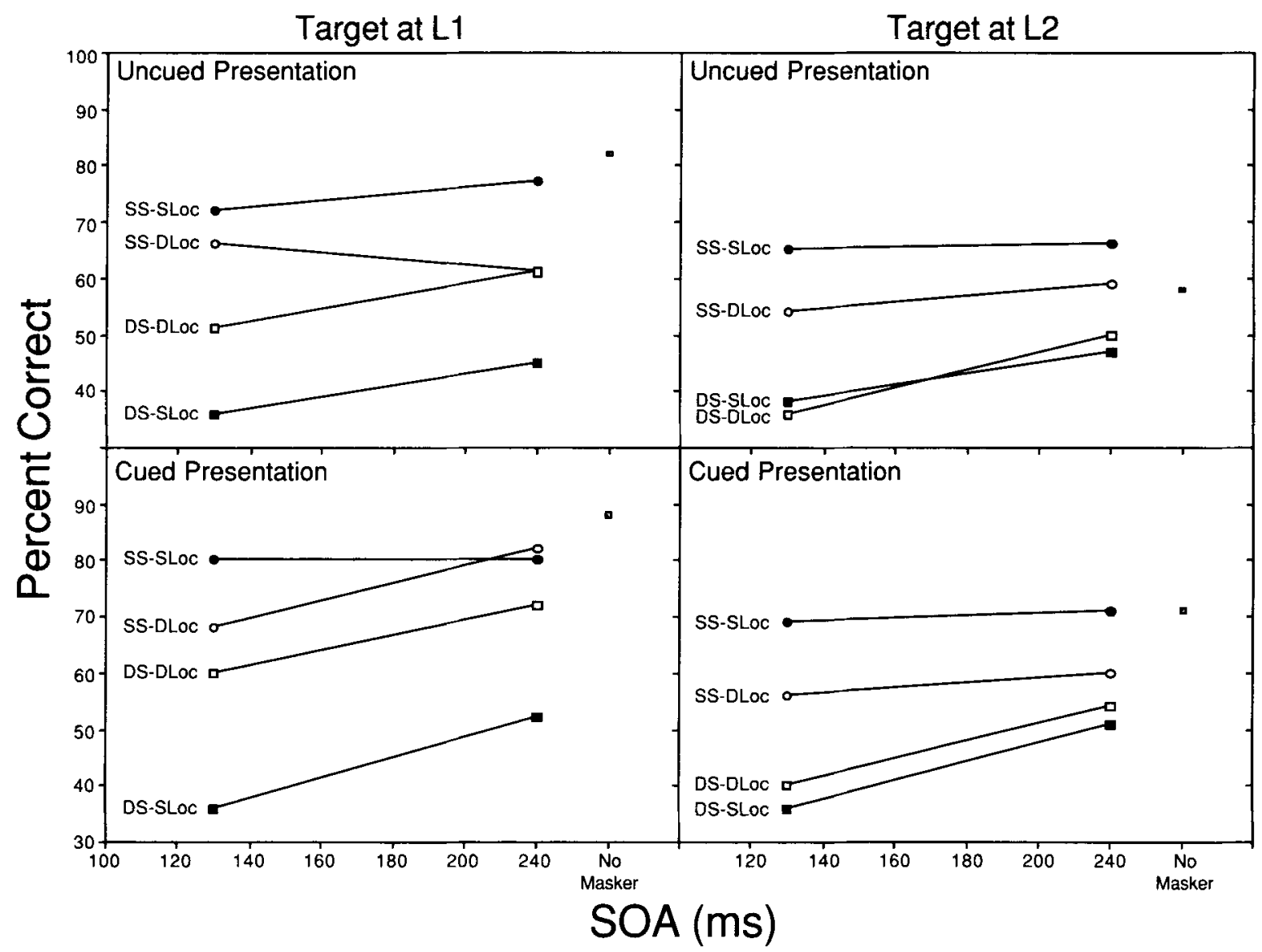

Figure 4. Percentage of correct target responses as a function of stimulus onset asynchrony (SOA) in the uncued presentation condition (top panels) and in the cued presentation condition (bottom panels). Left panels show performance when targets were at $L 1$ and right panels show performance when targets were at L2. The functions in each panel show performance when SS maskers were at SLoc (SS-SLoc), when SS maskers were at DLoc (SS-DLoc), when DS maskers were at SLoc (DS-SLoc), and when DS maskers were at DLoc (DS-DLoc). L, location; SS, same shape; DS, different shape. 
Comparing the uncued data in Figure 4 with the data in Figure 2 reveals similar results across different observers, patterns, durations, and SOAs. The similarity of these findings is particularly surprising given that observers in Experiment 2 were naive, but those in Experiment 1 were practiced. In addition, any advantage in reducing the set size from four patterns in Experiment 1 to three patterns in Experiment 2 appears to have been offset by the reduction in the physical size of the patterns in Experiment 2 relative to those in Experiment 1 . Reducing pattern size lowers recognition performance (Bliss, 1969; Johnson \& Phillips, 1981; Loomis, 1980, 1981, 1985; Phillips, Johnson, \& Browne, 1983).

The uncued data are also similar to the cued data, indicating that foreknowledge of the target's location had little effect on the pattern of interference by SS or DS maskers at SLoc or DLoc. The data in each of the panels of Figure 4 were analyzed using separate ANOVAs, and the results were similar for cued and uncued presentation. All four data sets had significant main effects of masker shape and SOA: As in Experiment 1, PC was lower in the presence of DS than of SS maskers, and PC improved with increasing SOA. Although there were also significant interactions between masker shape and masker location in all four data sets, the nature of these interactions differed for the $\mathrm{L} 1$ and $\mathrm{L} 2$ data. These interactions are discussed separately for uncued and cued presentation in the following sections.

Uncued presentation. As noted, uncued performance was similar to that in Experiment 1. When targets were at L1, DS maskers interfered more at SLoc than at DLoc, and SS maskers interfered more at DLoc than at SLoc. The small decline in performance at the longest SOA when SS maskers were at DLoc was not significant (Tukey HSD test, $p>.05$ ). When targets were at L2, DS maskers produced similar interference at SLoc and at DLoc, but unlike in Experiment 1, varying the location of SS maskers affected performance. SS maskers at DLoc caused little interference, but performance when SS maskers were at SLoc was higher than in the absence of maskers $[F(1,5)=7.58, p<.05]$, perhaps because an initially weak representation of the target fading over time may have been strengthened by the arrival of an identical masker at the same location (effectively extending processing time).

One might argue that response selection could also explain why target identification at L2 was enhanced by SS maskers at SLoc: Observers may have selected the strongest response from among the target and masker, increasing the likelihood of a correct response. However, such an explanation would predict even better performance at L2 in the presence of SS maskers at DLoc, since these maskers would be at $\mathrm{L} 1$ and presumably easier to recognize. Another prediction based on response selection is that the proportions of masker responses should differ from those in Experiment 1, in which there was no advantage in target identification at L2 when SS maskers were at SLoc. Because only three patterns were used in
Experiment 2, the proportion of masker responses on incorrect trials would have been $50 \%$ by chance. The proportion of masker responses averaged across SOAs was $68 \%$ when maskers were at $\mathrm{L} 1$ and $62 \%$ when maskers were at $\mathbf{L} 2$. Observers responded with maskers more often than chance and slightly more often when maskers were at $\mathrm{L} 1$ than at $\mathrm{L} 2$. Thus, there was a similarity in masker responses between Experiments 1 and 2 .

To examine the role of response selection in more detail, masker responses were analyzed separately for trials in which targets were at $\mathrm{L} 2$. When targets were at $\mathrm{L} 2$, the proportion of masker responses was $60 \%$ when maskers were at $\mathrm{L} 1$ and $61 \%$ when maskers were at $\mathrm{L} 2$. The fact that observers responded equally often with maskers at $\mathrm{L} 1$ and $\mathrm{L} 2$ when targets were at $\mathrm{L} 2$ indicates that masker responses did not enhance accuracy at L2. In Experiment 1, when targets were at $\mathrm{L} 2$, the proportion of masker responses was $49 \%$ when maskers were at L1 and $50 \%$ when maskers were at L2. Thus, when identifying targets at L2, observers in both experiments responded with maskers about equally often when maskers were at $\mathrm{L} 1$ and $\mathrm{L} 2$. The fact that performance differed between experiments, but response tendencies did not, suggests that response selection did not cause the advantage in target identification at L2 in the presence of SS maskers at SLoc.

Cued presentation. For targets at $\mathrm{L} 1$, the general pattern of interference from maskers was similar to that for uncued presentation, although performance was higher, particularly at the long SOA. For targets at L2, DS maskers produced similar interference at SLoc and DLoc, as in the uncued condition, but SS maskers affected performance differently than in the uncued condition. Target identification at L2 was not enhanced by SS maskers at SLoc, as in the uncued condition $(p>.05)$; rather, there was little interference. It is difficult to compare the effects of SS maskers between cuing conditions since performance at L2 was higher in the cued condition, and there may have been little advantage in the arrival of an identical masker at the same location.

Another difference from the uncued condition was that performance at L2 was reduced by SS maskers at DLoc $[F(1,5)=11.13, p<.05]$. It seems unlikely that this reduction was due to responding with SS maskers at DLoc, since the maskers would be at L1 and thus relatively easy to recognize, producing correct responses. Furthermore, when targets were at L2, the proportion of masker responses was $54 \%$ when maskers were at $\mathrm{L} 1$ and $66 \%$ when maskers were at L2. Thus, there was little overresponding with maskers at DLoc. Similar results occurred when targets were at L1; the proportion of masker responses was $75 \%$ when maskers were at $\mathrm{L} 1$, but only $51 \%$ when maskers were at $\mathrm{L} 2$. With targets at $\mathrm{L} 1$, observers did not overrespond with maskers at DLoc, suggesting that interference by maskers at DLoc was not due to response competition. The results suggest that observers narrowed attention to the target location and avoided responding with maskers at DLoc. However, 
maskers at DLoc continued to interfere with target identification, perhaps due to lateral masking. Lateral masking may be less effective at long SOAs, leading to reduced interference.

The interference by maskers at DLoc differed for SS and DS maskers, suggesting that lateral masking may depend on the form of the masker. This is consistent with a finding by Loomis (1993) that lateral masking of tactile characters increased as a surround changed from solid lines to dots. Although it is difficult to compare Loomis's result with the findings of the present study because of differences in stimuli and procedure, it appears that the form of an adjacent stimulus may affect the degree to which it interferes with pattern perception. Furthermore, the effect of shape on lateral masking is unlikely to be due to temporal integration of target and masker features. To ensure this, stimulus-response (SR) matrices were examined for targets presented to each location in the absence of maskers and in the presence of SS or DS maskers at SLoc or DLoc.

In the absence of maskers, accuracy was about equal for all three patterns at $\mathrm{L} 1$, but there was a slight accuracy advantage for the $V$-shaped pattern at $L 2$. With the exception of more errors, similar patterns of responding occurred in the presence of SS maskers at SLoc or DLoc, and in the presence of DS maskers at DLoc. However, SR matrices were different in the presence of DS maskers at SLoc: Accuracy differed among patterns at L1, and the accuracy advantage for the V-shaped pattern was eliminated at $\mathrm{L} 2$. The fact that the pattern of responding changed when DS maskers were at SLoc, but not when SS maskers were at SLoc, is consistent with the notion of temporal integration of target and masker features. Such integration would lead to increased interference by DS maskers, but little interference by SS maskers. However, the pattern of responding was unchanged in the presence of SS and DS maskers at DLoc, suggesting that temporal integration did not cause the observed interference.

The preceding SR analysis has important implications for the difference in interference by SS and DS maskers at SLoc. It has often been assumed that temporal integration is responsible for the lack of interference by SS maskers at SLoc. However, if the shape of an adjacent masker partly determines the amount of lateral masking (in the absence of temporal integration), temporal integration may not be solely responsible for the different effects of SS and DS maskers at SLoc.

\section{GENERAL DISCUSSION}

The results suggest that both masking and response competition may contribute to the effects of location on identification under certain circumstances. During uncued presentation, observers overresponded with the masker regardless of its location, and thus response competition was likely to have produced interference. Responding with a DS masker should lead to an incorrect response unless observers misidentify the masker and by chance give the target response instead. Responding with an SS masker at SLoc should produce a correct response unless observers misidentify the masker. However, responding with an SS masker at DLoc may not always produce a correct response if SS patterns at DLoc are perceived to be different shapes (Horner, 1995). The interference by DS maskers at SLoc may have been due to a combination of response competition and temporal integration, explaining why performance was so low. Temporal integration and selecting masker responses would also explain the minimal interference by SS maskers at SLoc.

The fact that observers overresponded with maskers during uncued presentation suggests that they may have used a wide attentional spotlight. Attending to a large area would seem reasonable when the locations of anticipated targets are unknown, as might occur when observers are exploring an object for surface features. There may be an advantage in having attentional resources available to process features regardless of their locations on the skin, at least over the limited extent of the fingerpad. However, there may be at least one disadvantage - possible interference by adjacent features.

The fact that cued presentation nearly eliminated overresponding with maskers at DLoc suggests that observers can selectively attend to a single location on the fingerpad, although perhaps not perfectly. Cued performance was generally higher than was uncued performance, also suggesting that observers were able to attend selectively to a single location. With a priori knowledge of the target's location, observers may narrow their attentional spotlight to enhance target processing at a specific location. When observers are exploring the surface of an object, repositioning the fingerpad on a previously discovered surface feature may allow them to direct attention to a specific feature. However, despite the increased allocation of attention to a specific location, there may still be lateral masking from adjacent features. The absence of lateral masking and the ability to direct attention may contribute to success in reading with the Optacon or using braille; patterns scroll across the fingerpad at a known location in the absence of stimuli at adjacent locations.

Overall, the results indicated that location is important for perception of tactile spatial patterns. As expected, when DS patterns were presented, there was more interference when patterns were at identical than at different locations (Weisenberger, 1981). However, when SS patterns were presented, the reverse was true; there was more interference when patterns were at different than at identical locations. In short, the same shape presented to a different location interfered as if it were a different shape. This result is consistent with the finding that SS patterns presented to separate locations on a single fingerpad often feel like DS patterns (Horner, 1995). The identification results of the present study extend the previous 
discrimination results and suggest that shape and location are important features of tactile spatial patterns, at least on the fingerpad.

Interpretation of the results was complicated by the difference in accuracy at L1 and L2. It was suggested that this difference in performance was due to different densities of innervation at the two locations. One way to eliminate differences in accuracy might be to present patterns on the middle rather than distal phalange, where varying location would not be associated with different innervation densities (Johansson \& Vallbo, 1979; Vallbo \& Johansson, 1984). Plans are currently under way in the laboratory to test locations on the middle phalange.

Temporal masking is often mentioned as a primary limiting factor in using the skin to process successive patterns at a single location (Craig, 1976, 1978, 1983, 1985b). This translates into reduced reading rates for users of braille and the Optacon, in which rapid processing of successive patterns is of paramount importance. Aside from reducing the rate of pattern presentation, which would reduce reading rates, overcoming temporal masking has met with only partial success. Patterns have been presented to separate fingers to avoid masking, but performance is limited by attentional constraints (Craig, 1985a). The present results indicate that presenting successive patterns to separate locations on the same finger is unlikely to overcome temporal masking. Interference remains when patterns are presented to DLoc, and in the case of SS patterns, interference actually increases. The additional problem of differences in accuracy between locations may be eliminated by using the middle phalange, but accuracy would be reduced due to lower innervation density (Johansson \& Vallbo, 1979; Vallbo \& Johansson, 1984).

\section{REFERENCES}

BLISS, J. C. (1969). A relatively high-resolution reading aid for the blind. IEEE Transactions on Man-Machine Systems, 10, 1-9.

Bliss, J. C., Katcher, M. H., Rogers, C. H., \& Shepard, R. P. (1970). Optical-to-tactile image conversion for the blind. IEEE Transactions on Man-Machine Systems, 11, 58-64.

BLISS, J. C., \& LiNviLL, J. G. (1966). A direct translation reading aid: Reading alphabetic shapes tactually. In R. Dufton (Ed.), Proceedings of the International Conference on Sensory Devices for the Blind (pp. 389-407). London: Arrowsmith.

CAMPBELL, D. T., \& FISKE, D. W. (1959). Convergent and discriminant validation by the multitrait-multimethod matrix. Psychological Bulletin, 56, 81-105.

Cholewiak, R. W., \& Craig, J. C. (1984). Vibrotactile pattern recognition and discrimination at several body sites. Perception \& Psychophysics, 35, 503-514.

CRAIG, J. C. (1976). Vibrotactile letter recognition: The effects of a masking stimulus. Perception \& Psychophysics, 20, 317-326.

CraIG, J. C. (1977). Vibrotactile pattern perception: Extraordinary observers. Science, 196, 450-452.

CraIG, J. C. (1978). Vibrotactile pattern recognition and masking. In $\mathrm{G}$. Gordon (Ed.), Active touch-The mechanism of recognition of objects by manipulation: A multi-disciplinary approach (pp. 229-242). Oxford: Permagon.

CRaIG, J. C. (1980). Modes of vibrotactile pattern perception. Journal of Experimental Psychology: Human Perception \& Performance, 6 , 151-166.
CRAIG, J. C. (1982). Vibrotactile masking: A comparison of energy and pattern maskers. Perception \& Psychophysics, 31, 523-529.

CRaIG, J. C. (1983). The role of onset in the perception of sequentially presented vibrotactile patterns. Perception \& Psychophysics, 34, 421-432.

Craig, J. C. (1985a). Attending to two fingers: Two hands are better than one. Perception \& Psychophysics, 38, 496-511.

CRaIG, J. C. (1985b). Tactile pattern perception and its perturbations. Journal of the Acoustical Society of America, 77, 238-246.

CraIG, J. C. (1989). Interference in localizing tactile stimuli. Perception \& Psychophysics, 45, 343-355.

CraIG, J. C. (1995). Vibrotactile masking: The role of response competition. Perception \& Psychophysics, 57, 1190-1200.

Craig, J. C., \& Evans, P. M. (1987). Vibrotactile masking and the persistence of tactual features. Perception \& Psychophysics, 42, 309317.

Craig, J. C., \& Evans, P. M. (1995). Tactile selective attention and temporal masking. Perception \& Psychophysics, 57, 511-518.

DrIVER, J., \& BAYLIS, G. C. (1991). Target-distractor separation and feature integration in visual attention to letters. Acta Psychologica, 76, 101-119.

Eriksen, C. W., \& Hoffman, J. E. (1973). The extent of processing of noise elements during selective encoding from visual displays. Perception \& Psychophysics, 14, 155-160.

Evans, P. M. (1987). Vibrotactile masking: Temporal integration, persistence, and strengths of representations. Perception \& Psychophysics, 42, 515-525.

Evans, P. M., \& Craig, J. C. (1986). Temporal integration and vibrotactile backward masking. Journal of Experimental Psychology: Human Perception \& Performance, 12, 160-168.

Evans, P. M., \& Craig, J. C. (1991). Tactile attention and the perception of moving tactile stimuli. Perception \& Psychophysics, 49, 355364.

Evans, P. M., \& Craig, J. C. (1992). Response competition: A major source of interference in a tactile identification task. Perception \& Psychophysics, 51, 199-206.

Evans, P. M., Craig, J. C., \& Rinker, M. A. (1992). Perceptual processing of adjacent and nonadjacent tactile nontargets. Perception \& Psychophysics, 52, 571-581.

HILL, J. W. (1974). Limited field of view in reading lettershapes with the fingers. In F. A. Geldard (Ed.), Cutaneous communication systems and devices (pp. 95-105). Austin, TX: Psychonomic Society.

HoRNER, D. T. (1991). The effects of complexity on the perception of vibrotactile patterns. Perception \& Psychophysics, 49, 551-562.

HoRNer, D. T. (1992). The effects of complexity on the perception of vibrotactile patterns presented to separate fingers. Perception \& Psychophysics, 52, 201-210.

HORNER, D. T. (1995). The effect of location on the discrimination of spatial vibrotactile patterns. Perception \& Psychophysics, 57, 463474.

Horner, D. T., \& Craig, J. C. (1989). A comparison of discrimination and identification of vibrotactile patterns. Perception \& Psychophysics, 45, 21-30.

Johansson, R. S., \& Vallbo, A. B. (1979). Tactile sensibility in the human hand: Relative and absolute densities of the four types of mechanoreceptive units in glabrous skin. Journal of Physiology, 286, 283-300.

Johnson, K. O., \& Phillips, J. R. (1981). Tactile spatial resolution. I. Two-point discrimination, gap detection, grating resolution, and letter recognition. Journal of Neurophysiology, 46, 1177-1191.

Loomis, J. M. (1980). Interaction of display mode and character size in vibrotactile letter recognition. Bulletin of the Psychonomic Society, 16, 385-387.

Loomis, J. M. (1981). On the tangibility of letters and braille. Perception \& Psychophysics, 29, 37-46.

Loomis, J. M. (1985). Tactile recognition of raised characters: A parametric study. Bulletin of the Psychonomic Society, 23, 18-20.

Loomis, J. M. (1990). A model of character recognition and legibility. Journal of Experimental Psychology: Human Perception \& Performance, 16, 106-120.

Loomis, J. M. (1993). Counterexample to the hypothesis of functional 
similarity between tactile and visual pattern perception. Perception \& Psychophysics, 54, 179-184.

LOOMis, J. M., \& APKARIan-STIELAU, P. (1976). A lateral masking effect in tactile and blurred visual letter recognition. Perception \& Psychophysics, 20, 221-226.

Nolan, C. Y., \& Kederis, C. J. (1969), Perceptual factors in braille word recognition. New York: American Foundation for the Blind.

Phillips, J. R., Johnson, K. O., \& Browne, H. M. (1983). A comparison of visual and two modes of tactual letter resolution. Perception \& Psychophysics, 34, 243-249.

RinKer, M. A., \& Craig, J. C. (1994). The effect of spatial orientation on the perception of moving tactile stimuli. Perception \& Psychophysics, 56, 356-362.

Sathian, K., \& BURTON, H. (1991). The role of spatially selective attention in the tactile perception of texture. Perception \& Psychophysics, 50, 237-248.

Sathian, K., \& Zangaledze, A. (1996). Tactile spatial acuity at the human fingertip and lip: Bilateral symmetry and interdigit variability. Neurology, 46, 1464-1466.

SCADDEN, L. A. (1973). Tactile pattern recognition and body loci. Perception, 2, 333-336.

SherRick, C. E., \& Cholewiak, R. W. (1986). Cutaneous sensitivity. In K. R. Boff, L. Kaufman, \& J. P. Thomas (Eds.), Handbook of perception and human performance: Vol. II. Cognitive processes and performance (pp. 12-1 to 12-58). New York: Wiley.
Stevens, J. C., \& Patterson, M. Q. (1995). Dimensions of spatial acuity in the touch sense: Changes over the lifespan. Somatosensory Motor Research, 12, 29-47.

Vallbo, A. B., \& Johansson, R. S. (1984). Properties of cutaneous mechanoreceptors in the human hand related to touch sensation. Human Neurobiology, 3, 3-14.

VAN Boven, R. W., \& JohnSON, K. O. (1994). The limit of tactile spatial resolution in humans: Grating orientation disrimination at the lip, tongue, and finger. Neurology, 44, 2361-2366.

WEINSTEIN, S. (1968). Intensive and extensive aspects of tactile sensitivity as a function of body part, sex, and laterality. In D. R. Kenshalo (Ed.), The skin senses (pp. 195-222). Springfield, IL: Thon .

WEISENBERGER, J. M. (1981). Tactile pattern similarity. Unpublished doctoral dissertation, Indiana University, Bloomington.

WeISENBERGER, J. M., \& Craig, J. C. (1982). A tactile metacontrast effect. Perception \& Psychophysics, 31, 530-536.

Whang, K. C., Burton, H., \& Shulman, G. L. (1991). Selective attention in vibrotactile tasks: Detecting the presence and absence of amplitude change. Perception \& Psychophysics, 50, 157-165.

(Manuscript received August 22, 1996; revision accepted for publication December $8,1996$. 